Article

\title{
Sunitinib-Loaded MPEG-PCL Micelles for the Treatment of Age-Related Macular Degeneration
}

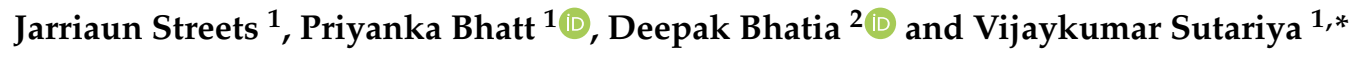 \\ 1 Department of Pharmaceutical Sciences, Taneja College of Pharmacy, University of South Florida, \\ 12901 Bruce B. Downs Blvd., MDC30, Tampa, FL 33612, USA; jstreets@usf.edu (J.S.); psbhatt@usf.edu (P.B.) \\ 2 Department of Pharmacogenomics, Bernard J Dunn School of Pharmacy, Shenandoah University, \\ 8095 Innovation Park Drive, Fairfax, VA 22031-4868, USA; dbhatia@su.edu \\ * Correspondence: vsutariy@usf.edu
}

Received: 1 June 2020; Accepted: 20 July 2020; Published: 22 July 2020

\begin{abstract}
Age-related macular degeneration (AMD) will be responsible for the vision impairment of more than five million late-aged adults in the next 30 years. Current treatment includes frequent intravitreal injections of anti-vascular endothelial growth factor (VEGF) agents. However, there are methods of drug delivery that can decrease the frequency of intravitreal injections by sustaining drug release. MPEG-PCL ((methoxypoly(ethylene glycol) poly(caprolactone)) has been reported as biocompatible and biodegradable. Polymeric micelles of MPEG-PCL can be useful in efficiently delivering anti-VEGF drugs such as sunitinib to the posterior segment of the eye. In this study, the novel micellar formulation exhibited an average dynamic light scattering (DLS) particle size of $134.2 \pm 2.3 \mathrm{~nm}$ with a zeta potential of $-0.159 \pm 0.07 \mathrm{mV}$. TEM imaging further confirmed the nanoscopic size of the micelles. A sunitinib malate (SM)-MPEG-PCL formulation exhibited a sustained release profile for up to seven days with an overall release percentage of $95.56 \pm 2.7 \%$. In addition to their miniscule size, the SM-MPEG-PCL formulation showed minimal cytotoxicity onto the ARPE-19 human retinal pigment epithelial cell line, reporting a percent viability of more than $88 \%$ for all concentrations tested at time intervals of $24 \mathrm{~h}$. The SM-MPEG-PCL micelles also exhibited exceptional performance during an anti-VEGF ELISA that decreased the overall VEGF protein expression in the cells across a $24-72 \mathrm{~h}$ period. Furthermore, it can be concluded that this type of polymeric vehicle is a promising solution to symptoms caused by AMD and improving the management of those suffering from AMD.
\end{abstract}

Keywords: sunitinib; micelles; age-related macular degeneration; sustained release; anti-VEGF; MPEG-PCL

\section{Introduction}

Age-related macular degeneration (AMD) is an eye disease that gradually deteriorates the macula and is the leading causes of vision failure among late-aged adults [1,2]. The macula is located in the posterior segment of the eye near the retinal center, and it is a key component for central vision processing by using its photoreceptor cells [3]. Macular degradation as a result of AMD disrupts the process of normal vision processing in the optic nerve, thus causing blurry and distorted vision. It is projected that by 2050, more than five million Americans will have some form of AMD [1]. Vascular endothelial growth factor (VEGF) is a protein that plays a major role in the formation of blood vessels within the eye in neovascular AMD. Anti-VEGF drugs inhibit the expression of VEGF at the angiogenic site to prevent central vision damage induced by hyperoxic conditions [4]. Generally, anti-VEGF drugs such as axitinib, pazopanib, and sorafenib are available for use in cancer treatments, but they recently have been propitious agents for treating AMD-induced neovascularization [5,6]. Despite the success of 
these angiogenic inhibitors, a newer drug named sunitinib malate has proven to be a notable contender for the treatment of ocular diseases $[7,8]$.

Sunitinib malate (SM) is a tyrosine kinase inhibitor that exhibits anti-VEGF and anti-HIF properties [9]. Originally approved for the treatment of renal cell carcinoma, sunitinib has displayed usefulness in the treatment of ocular diseases like AMD that depend on VEGF or platelet-derived growth factor (PDGFR) receptors [10]. Sunitinib can specifically inhibit all three types of VEGF receptors (VEGFR1-VEGFR3), along with seven other important components of cell proliferation. Notably, VEGFR1, VEGFR2, and PDGFR (beta) are the main integrants of angiogenesis in the body. Additionally, sunitinib can inhibit eight total tyrosine kinase receptor proteins, such as Kit, Flt-3, and CSF-1R [11]. Because sunitinib is able to terminate tyrosine kinase binding, it can potentially inhibit ocular angiogenesis, a common symptom of AMD [12]. Nonetheless, it is noted that the use of sunitinib is restricted due to its toxicity among higher dosages $[7,8]$.

However, there are significant physical barriers including the blood-retinal barrier to reaching the posterior segment of the eye where the retina and macula are housed [13-15]. Because of this, patients treated for AMD are subject to painful and frequent intravitreal injections that lead to a low patient compliance. However, poly(ethylene glycol)-block-poly ( $\varepsilon$-caprolactone) (PEG-PCL) copolymers have been recognized for their low toxicity, biocompatibility, and ability to aid in sustained drug release [16]. PEG, along with PEG derivatives like PEG-PCL have been approved by the FDA for medical uses [17]. MPEG-PCL (methoxypoly(ethylene glycol) poly(caprolactone)) copolymers are widely researched and notable synthetic material of biomedical and drug delivery applications due to their controlled biodegradable nature and amphiphilicity, as well as their biocompatible properties. Their wide applications include fields such as tissue engineering, nanotechnology, pharmaceutics, and medicinal chemistry. MPEG-PCL copolymers have a very low glass transition temperature and are semi-crystalline in nature. They are ideal polymers to be used for designing sustained delivery formulations to deliver an entrapped therapeutic moiety for longer periods due to their slow degradation. In contrast to MPEG-PCL, the PEG-PCL copolymer, which has short PCL blocks, retains the crystalline structure of PEG. However, it was reported that MPEG-PCL copolymers with long PCL chains present the same crystalline structure as PCL homopolymers [18]. The optimal biodistribution of PEG-PCL nanoparticles was observed in vivo and ex vivo studies using parameters between 20 and $200 \mathrm{~nm}$ in size and molecules with a negative zeta potential, thus indicating that the circulation time of drugs carried by PEG-PCL copolymers can be greatly extended by reducing the frequency of administration [19]. PEG-PCL micelles for the delivery of the anti-VEGF drug axitinib also expressed their usefulness in inhibiting angiogenesis while displaying exceptional cytocompatibility $[20,21]$. These advancements are promising in fulfilling the need to decrease frequent intravitreal injections and to significantly improve patient compliance [22-24].

Consequently, MPEG-PCL micelles, in conjunction with sunitinib malate, appears to be a viable option due to its ability to inhibit the eight receptors in the body responsible for cell proliferation and angiogenesis when administered intravitreally [21,25]. The objective of this investigation was to formulate sustained release formulation SM-MPEG-PCL micelles and to evaluate the formulation using physiochemical methods and cell-based assays.

\section{Materials and Methods}

\subsection{Materials}

Sunitinib malate was purchased from Selleck Chemicals, Houston, TX, USA. All (2-2k, 5-2k, and 5-10k) methoxy poly(ethylene glycol)-block-poly ( $\varepsilon$-caprolactone) polymers were purchased from Sigma-Aldrich, St. Louis, MO, USA. Organic solvents, such as methanol, ethanol, dimethyl sulfoxide (DMSO), and acetone were purchased from Thermo Fischer Scientific, Waltham, MA, USA. The dialysis membrane (Mw 14000) was purchased from Sigma Aldrich (St. Louis, MO, USA). Cell culturing materials such as fetal bovine serum and streptomycin-penicillin antibiotic $(10,000 \mathrm{U} / \mathrm{mL})$ were 
purchased from Gibco Thermo Fischer Scientific, USA. ARPE-19 (American Type Culture Collection (ATCC)(C CRL2302 ${ }^{\mathrm{TM}}$ ). A human retinal pigment epithelial cell line, along with a DMEM F12 medium, was purchased from ATCC (VA, USA). Trypsin $(0.05 \%)$ was obtained from Thermo Fischer Scientific (Lansing, MI, USA), whereas a $1 \times$ phosphate-buffered saline solution (PBS) was obtained from Corning Cellgro (Manassas, VA, USA). An MTT (3-(4,5 dimethylthiazol-2-yr)-2,5-diphenyltetrazolium) bromide salt reagent was purchased from Sigma Aldrich (St. Louis, MO, USA). An Invitrogen ${ }^{\mathrm{TM}}$ eBioscience $^{\mathrm{TM}}$ Human VEGF ELISA Kit was purchased from Fischer Scientific (Lansing, MI, USA).

\subsection{Cell Culture}

Cell line studies were carried out using ARPE-19 (ATCC C CRL2302 ${ }^{\mathrm{TM}}$ ) cells derived from a human retinal epithelial cell line. Nourishment for the cells was provided from complete media, a combination of a DMEM F12 medium, 10\% $v / v$ fetal bovine serum (FBS), and 1\% 10,000 $\mu / \mathrm{mL}$ penicillin antibiotic. Additionally, cells were stored in a moisture-controlled $5 \% \mathrm{CO}_{2}$ incubator set to $37^{\circ} \mathrm{C}$.

\subsection{Formulation of SM-MPEG-PCL Micelles}

Two milligrams of sunitinib malate were dissolved in $200 \mu \mathrm{L}$ of DMSO into a $5 \mathrm{~mL}$ centrifuge tube. This mixture was sonicated for eight minutes to achieve the full dissolution of the drug into the organic solvent. Afterwards, $1.8 \mathrm{~mL}$ of acetone along with $20 \mathrm{mg}$ of the MPEG-PCL (5-2k) polymer were added to the DMSO/sunitinib mixture and sonicated again to fully dissolve the drug. The mixture was then vortexed to mix the two solvents; this was the $2 \mathrm{~mL}$ organic solvent component. The accompanying aqueous phase was $4 \mathrm{~mL}$ of filtered distilled water. Under magnetic stirring, the $2 \mathrm{~mL}$ organic phase was added dropwise using a 23G syringe to the aqueous phase. The mixture was then stirred magnetically for $24 \mathrm{~h}$ at $700 \mathrm{rpm}$ to allow for the adequate precipitation of organic solvents. After $24 \mathrm{~h}$, the formulation was then centrifuged at $5000 \mathrm{rpm}$ for $15 \mathrm{~min}$ to extrapolate any free drugs or polymers, as seen in Figure 1C.

A.

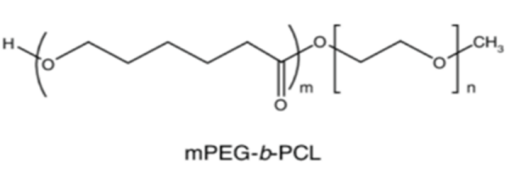

B.<smiles>CCN(CC)CCNC(=O)c1c(C)[nH]c(C=C2c3cc(F)ccc3NC2O)c1C</smiles>

C.
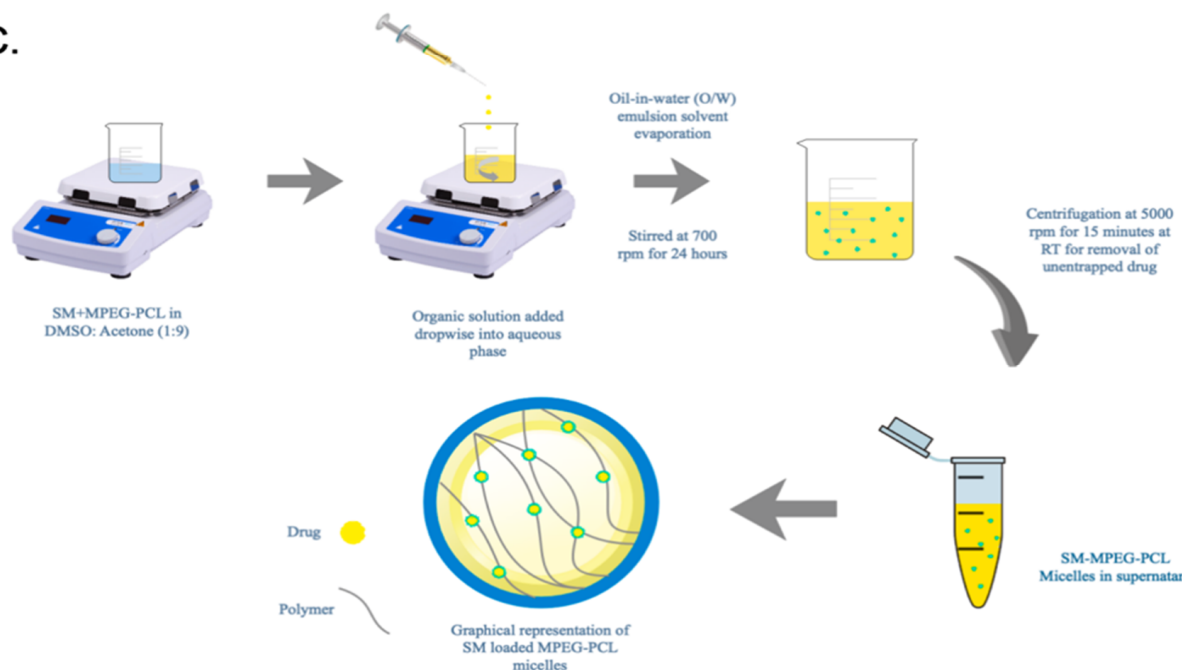

SM-MPEG-PCL

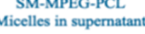

Figure 1. (A) Structure of the ((methoxypoly(ethylene glycol) poly(caprolactone)) (MPEG-PCL) polymer, $(\mathbf{B})$ the structure of sunitinib, and $(\mathbf{C})$ the preparation scheme for the sunitinib malate (SM)-MPEG-PCL micelles. 


\subsection{Differential Scanning Calorimetry}

Differential scanning calorimetry (DSC) analysis was executed using a DSC Q 20 instrument (TA Instruments, New Castle, DE, USA, Q series Q20-2288-DSC software). Four samples were analyzed: the polymer, the pure sunitinib drug, the physical mixture of sunitinib and the MPEG-PCL polymer, and the SM-MPEG-PCL formulation. Five milligrams of each sample were taken and placed inside of an aluminum pan specifically made for DSC analysis [26]. Samples were then heated to approximately $30-300^{\circ} \mathrm{C}$ at a rate of $10^{\circ} \mathrm{C}$ while simultaneously being under a nitrogen purge at a rate of $50 \mathrm{~mL} / \mathrm{min}$ [27].

\subsection{Particle Size and Zeta Potential}

The size and zeta potential of the SM-MPEG-PCL micelles were measured using dynamic light scattering (DLS) via the Zetasizer Nano ZS 90 instrument (Malvern Instruments Ltd., UK, Zetasizer Software Ver. 7.10). The parameters for characterizing nanomicelles included a 90 degree measuring angle along with $25^{\circ} \mathrm{C}$ temperature for each sample. Each cuvet consisted of $900 \mu \mathrm{L}$ of filtered distilled water and $100 \mu \mathrm{L}$ of the formulation [28]. The Zetasizer reported characterization data in triplicates, along with an average polydispersity index (PDI), which measures the biomolecular distribution of nanomicelles within a solution. The particle size and PDI of the formulation were also evaluated in various buffers, such as $0.01,0.1$, and $1 \mathrm{M}$ PBS.

Using a zeta cuvette cell, zeta potential was measured with a total of $1 \mathrm{~mL}$ of the sample diluted with filtered distilled water. Zeta potential utilizes the dynamics of the Smoluchowski equation, which electrically determines the motility of particles in conjunction with a back-scatter of $90^{\circ}$ [29]. Similar to size characterization, zeta potential data were also measured in triplicates.

\subsection{Transmission Electron Microscopy}

The morphology of the SM-MPEG-PCL micelles were measured using TEM. To prepare the formulation for imaging, the sample was placed onto a $200 \mathrm{Cu}$ film square grid. The sample was then allowed to settle for approximately $10 \mathrm{~min}$. After the sample was air dried, it was prepared for negative staining using $2 \% w / v$ phosphotungstic acid (PTA). Furthermore, images were obtained at $80,000 \times$ magnification at an accelerating voltage of $120 \mathrm{kV}$.

\subsection{Entrapment Efficiency}

The SM-MPEG-PCL formulation was centrifuged at $5000 \mathrm{rpm}$ for $15 \mathrm{~min}$ to separate any excess drugs or polymers. Furthermore, the supernatant was collected and centrifuged once more at 18,000 rpm for approximately $20 \mathrm{~min}$ to transform the micelles into pellet form for further analysis. In order to prepare the formulation for UV spectrophotometer analysis, the pellet was washed three times with filtered distilled water to further ensure the removal of the unentrapped drug. The nanoformulation was dissolved with methanol as a solvent and analyzed using a UV spectrophotometer at a wavelength of $432 \mathrm{~nm}(\lambda \max )$. From this data, the amount of drug within the sample was calculated and used in the equations below to determine the entrapment efficiency and loading efficiency percentages.

$$
\begin{gathered}
\% \text { Entrapment Efficiency }=\frac{\text { Actual amount of drug loaded in micelles }}{\text { Actual amount of drug used for preparation }} \times 100 \\
\% \text { Loading Efficiency }=\frac{\text { Amount of Sunitinib in micelles }}{\text { Total amount of micelles }} \times 100
\end{gathered}
$$

\subsection{In Vitro Drug Release Study}

The in vitro drug release included a comparison of the pure SM drug solution and the SM-MPEG-PCL formulation by the use of a dialysis bag (MWCO 14,000 Da) [30]. In preparation for the loading of both the sunitinib solution and the SM-MPEG-PCL formulation, the release medium 
was prepared by the addition of PBS with a pH adjusted to 7.4 [31], along with a Tween 80 solution $(0.1 \% v / v)$. The release medium was further optimized under magnetic stirring at $100 \mathrm{rpm}$ with a constant temperature of $37^{\circ} \mathrm{C}$. Out of the $5 \mathrm{~mL}$ of the release medium, approximately $200 \mu \mathrm{L}$ of each formulation were taken and subsequently replaced with $200 \mu \mathrm{L}$ of the release medium at time intervals of $0.25,0.5,1,2,3,4,5$, and $6 \mathrm{~h}$ for the initial day and every $24 \mathrm{~h}$ for the next seven days. Samples were diluted with methanol in preparation to be read using a UV spectrophotometer at a wavelength of $432 \mathrm{~nm}$.

\subsection{Cytotoxicity Study}

The cytotoxicity of the SM-MPEG-PCL formulation and the pure drug solution were measured using an MTT assay analysis on ARPE-19, a human retinal pigment epithelial cell line. In a 96-well plate, cells were seeded at a density of 5000 cells per well supplemented with a medium consisting of $200 \mu \mathrm{L}$ of DMEM F12 and a 10\% FBS solution. Cells were then allowed to grow in a 5\% atmosphere, $37^{\circ} \mathrm{C}, \mathrm{CO}_{2}$ incubator for $24 \mathrm{~h}$ to ensure the proper attachment of the cells to the bottom of the well plates [31]. Following initial incubation, cells were then treated with dilutions of $0.001,0.01,0.1,1,10$, and $20 \mu \mathrm{M}$ of the drug solution, as well as the SM-MPEG-PCL formulation in quadruplicate. Similarly, growth media and Triton X100 were used as positive and negative controls, respectively [32].

After treatment, cells were allowed to incubate for $4 \mathrm{~h}$. Subsequently, the treatments were removed, and cells were then washed once thoroughly with a sterile $1 \times$ PBS solution. Afterwards, cells were supplemented with a fresh complete medium and were incubated for 24 and $48 \mathrm{~h}$. After $24 \mathrm{~h}$, the complete media were removed from the cells and replaced with $100 \mu \mathrm{L}(1 \mathrm{mg} / 1 \mathrm{~mL})$ of MTT reagent before being incubated for $4 \mathrm{~h}$ at $37^{\circ} \mathrm{C}$ in a $5 \% \mathrm{CO}_{2}$ atmosphere. After $4 \mathrm{~h}$, the MTT reagent was removed and replaced with $100 \mu \mathrm{L}$ of DMSO to dissolve the formazan crystals that indicated cell viability by displaying a distinct purple color. The plate was then read using a UV spectrophotometer at a wavelength of $595 \mathrm{~nm}$ to obtain the absorbances of each well. For comparison, cells treated with DMEM-F12 were the negative control, and 0.1\% Triton X100 was the positive control.

\subsection{Anti-VEGF ELISA}

In a sterile 96-well plate, wells were seeded with a density of 5000 cells per well and treated with the complete media to induce confluency. After $24 \mathrm{~h}$, the complete medium was removed, and cells received treatment. Two treatments were used in the ELISA, beginning with free drug solution and ending with the SM-MPEG-PCL formulation. Complete and incomplete media were used as negative controls, and two treatments each at $10 \mu \mathrm{M}$ were treated four times for 24, 48, and $72 \mathrm{~h} \mathrm{[33].} \mathrm{After} 72 \mathrm{~h}$ of incubation, VEGF expression was assessed using an Invitrogen Human VEGF-A Platinum ELISA Kit. The quantification of cell protein content was measured using a Pierce BCA Protein Assay Kit following the collection of the cell lysate. The quantitative analysis of samples were read using an ELISA plate reader at an absorbance of $450 \mathrm{~nm}$. The differences between the two absorbances were noted and used to determine the inhibitory effects of VEGF secretion using a standard curve.

\subsection{Statistical Analysis}

In order to quantize all data reported in this study, an extensive statistical analysis was done using the mean, standard deviation, ANOVA, and Student's t-test. GraphPad Prism (Version 6, USA) was used for all analyses and $p$-values reported at levels of $<0.05,<0.01$, or $<0.001$ at each place.

\section{Results and Discussions}

\subsection{Differential Scanning Calorimetry}

The physicochemical properties of the pure SM drug, the MPEG-PCL polymer, the physical mixture of the MPEG-PCL polymer and the SM drug, and the SM-MPEG-PCL micelle formulation were analyzed using DSC analysis. Figure 2 displays the thermal analysis of each. The nature and 
physical state of the encapsulated drug in a polymer matrix can have effect on its release, and DSC thermograms thus enabled us to identify this nature. Sunitinib alone exhibited an endothermic peak at a temperature of $200.86^{\circ} \mathrm{C}$, indicative of its melting point and confirming purity of drug (Figure 2). That peak was absent in the DSC spectrum for the SM-MPEG-PCL micelle formulation, thus confirming the entrapment of SM in the formulation and the presence of SM as the amorphous form [34]. However, a characteristic endothermic peak of melting point of SM was present at $200.62{ }^{\circ} \mathrm{C}$ in the physical mixture of SM and the MPEG-PCL polymer, which showed that physical mixing was not optimum to entrap the drug in polymer matrix. The endothermic peak at $55^{\circ} \mathrm{C}$ in the spectra of the polymer and the physical mixture represents characteristic peak of the polymer itself.

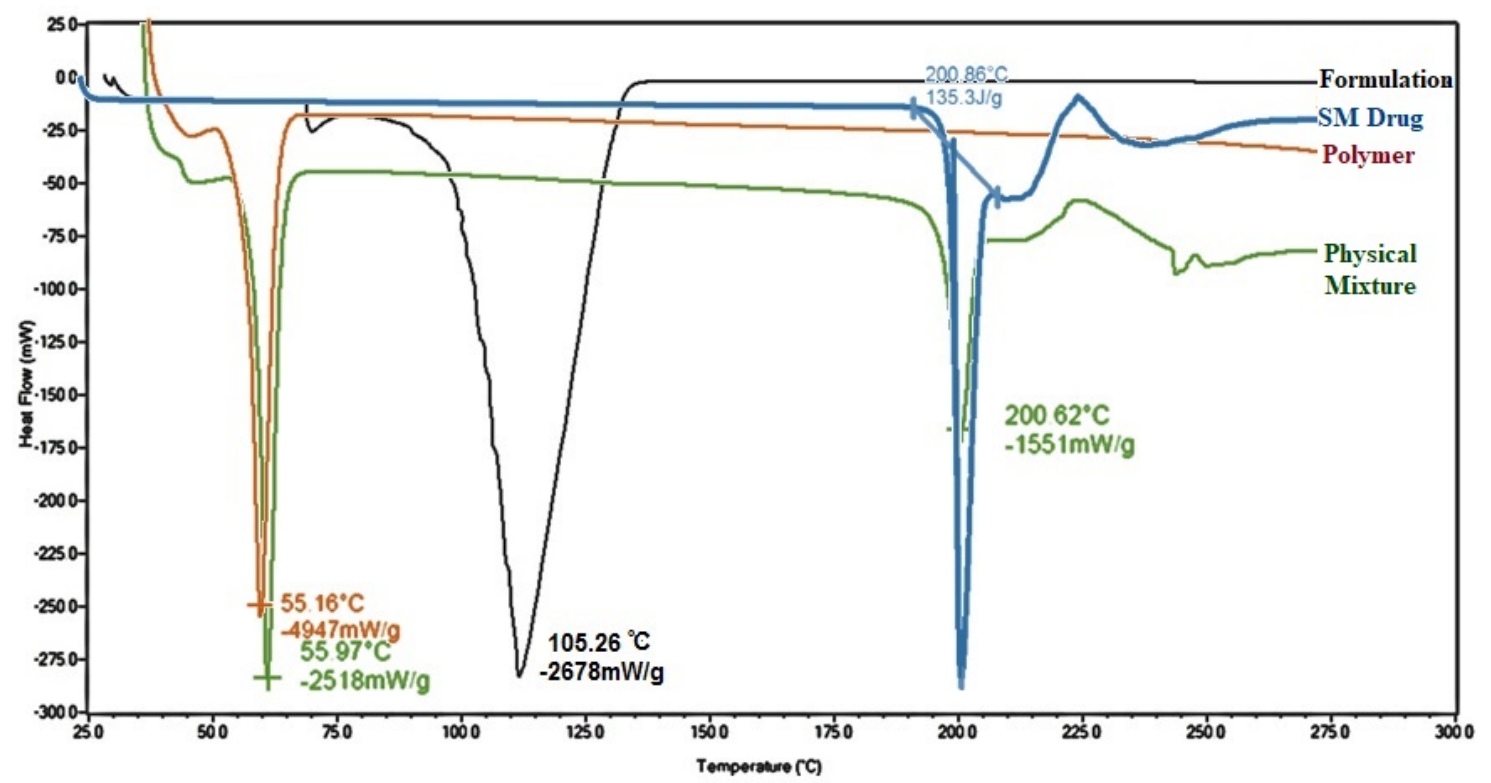

Figure 2. The differential scanning calorimetry (DSC) spectra of the formulation (SM-MPEG-PCL micelles), the SM drug (sunitinib malate pure drug), the polymer (MPEG-PCL polymer alone), and the physical mixture (the physical mixture of SM and MPEG-PCL).

\subsection{Particle Size and Zeta Potential}

From pre-formulation studies, it was concluded that the 1:10 (w/w) ratio of the MPEG-PCL (5-2k) polymer was the optimal molecular ratio to use for this experiment based on obtained results of size, PDI, zeta potential, and entrapment efficiency [35]. The particle size, along with the PDI of SM-MPEG-PCL, was determined with the dynamic light scattering technique and was measured to be of the order of $134.2 \pm 2.3 \mathrm{~nm}$; the PDI was $0.160 \pm 0.08$. The low PDI values indicated the homogeneity and monotonous distribution of the nanomicelles within the formulation [36]. The particle size of the formulation was also evaluated in $0.01,0.1$, and 1 M PBS to check the effect of various buffer systems on the particle size of the formulation. In contrast to the particle size of $134.2 \pm 2.3 \mathrm{~nm}$ and the PDI of $0.160 \pm 0.08$ in distilled water as a diluting medium, there was no drastic change in the size found after diluting formulation with different strengths of PBS buffers. Particle sizes of 193.2 $\pm 1.4,189.2 \pm 2.6$, and $185.1 \pm 3.1$ were measured for SM-MPEG-PCL in 0.01, 0.1, and 1 M PBS, respectively. Moreover, the PDI was found to be $0.220 \pm 0.06,0.196 \pm 0.08$, and $0.143 \pm 0.04$ for SM-MPEG-PCL in 0.01, 0.1, and 1 M PBS, respectively. Even after diluting in various buffers, the particle size of the formulation remained in optimum size less than $200 \mathrm{~nm}$ for effective ocular administration.

The mean zeta potential of SM-MPEG-PCL was determined using a Malvern Zetasizer Nano ZS90 and was $-0.159 \pm 0.07 \mathrm{mV}$. Figure $3 \mathrm{~A}$ shows the Z-average and the correlation functional plots for SM-MPEG-PCL. The MPEG-PCL micelles showed a negative zeta potential based on the surface charge on polymeric micelles, for which there could be many reasons such as the chemical structure of the MPEG-PCL polymer, the pH of the diluting medium, and the ionic strength of the medium. 
Another researcher also found similar results regarding zeta potential [37]. A negative zeta potential avoids the aggregation of nanoformulations and stabilizes them against electrostatic interaction forces. It also prevents the interaction of nanoformulations with serum components and protein adsorption to provide long systemic circulation.

A.

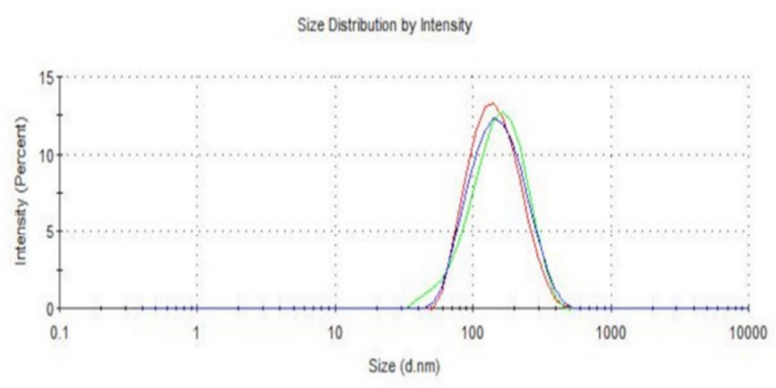

Record 125: 5K25THREE 1

Record 126: 5K25THREE 2

Record 127: 5K25THREE 3

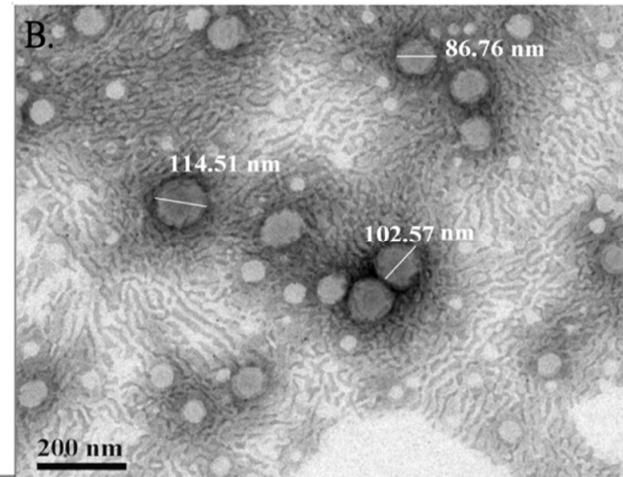

Figure 3. (A) Z-average of the SM-MPEG-PCL micelles and (B) 80,000× TEM image of the SM-MPEGPCL micelles at an accelerating voltage of $120 \mathrm{kV}$ (scale bar $200 \mathrm{~nm}$ ).

\subsection{Transmission Electron Microscopy}

TEM images reported particle sizes ranging from 86.76 to $114.51 \mathrm{~nm}$ in contrast to the mean particle size of $134.2 \mathrm{~nm}$ reported from DLS (Figure 3B). The discrepancy between the difference in particle sizes in DLS and TEM was due to the differences in the way the formulation was processed. TEM analyzed the nanomicelles within a fixed copper grid, whereas DLS utilized the hydrodynamic diameter of the micelles to report a mean particle size [38]. The TEM imaging of SM-MPEG-PCL micelles affirmed the size uniformity of the nanomicelles, coinciding with the low PDI of the 5-2k formulation. Figure 3B also shows the physical characteristics of the nanomicelles with a spherical structure and monotonous surface. It is also accurate to infer from the TEM imaging that the particles were in agreement with the negative zeta potential value and less likely to agglomerate.

\subsection{Entrapment Efficiency}

The SM-MPEG-PCL formulation was centrifuged at $5000 \mathrm{rpm}$ for $15 \mathrm{~min}$ to separate any excess drugs or polymers. Furthermore, the supernatant was collected and centrifuged once more at 18,000 rpm for approximately $20 \mathrm{~min}$ to transform the micelles into pellet form for further analysis. The micellar pellet was then collected and diluted with methanol to extract the entrapped drug. This dilution was then read at an $\lambda_{\text {max }}$ of $432 \mathrm{~nm}$ using a UV spectrophotometer and calculated according to absorbances obtained from the plate reader in conjunction with the previous equation $y=0.0458 \mathrm{x}-0.0035$ and the $r^{2}$ value of 0.999 of calibration curve of SM in methanol. The 1:10 (w/w) ratio of the 5-2k SM-MPEG-PCL formulation reported an entrapment efficiency of $64.3 \pm 2.6 \%$, whereas the entrapment efficiency for the $1: 15$ ratio was $45.6 \pm 1.8 \%$. Hence, the $1: 15$ ratio was not feasible to use for further studies despite its smaller particle size.

\subsection{In Vitro Drug Release}

The in vitro drug release study using the dialysis method compared the release profile of the SM drug solution and SM-MPEG-PCL over a seven-day period. The release medium was prepared using PBS ( $\mathrm{pH} 7.4)$ and Tween $80(0.1 \% v / v)$ at an approximate temperature of $37 \pm 2{ }^{\circ} \mathrm{C}$ for seven days. The drug solution exhibited a complete expulsion in the release media at approximately $12 \mathrm{~h}$ after initial administration. In contrast, the SM-MPEG-PCL formulation saw the full release of drug from the formulation at approximately 168 h, or seven days, as seen in Figure 4 . Because of this, we can infer that the MPEG-PCL formulation is capable sustaining the release of the drug for seven days. 
Within $2 \mathrm{~h}$ of the initiation, the SM formulation displayed a burst release of $11.8 \pm 1.2 \%$. Meanwhile, after $24 \mathrm{~h}$, the formulation had a SM release of $39.44 \pm 4.1 \%$. Furthermore, the formulation displayed a sustained release profile of $95.56 \pm 2.7 \%$ of SM from the formulation after the seven-day period. Hypothetically, this can be classified as sustained drug release, but the further optimization of the formulation is needed to extend the release of the drug for longer than seven days.

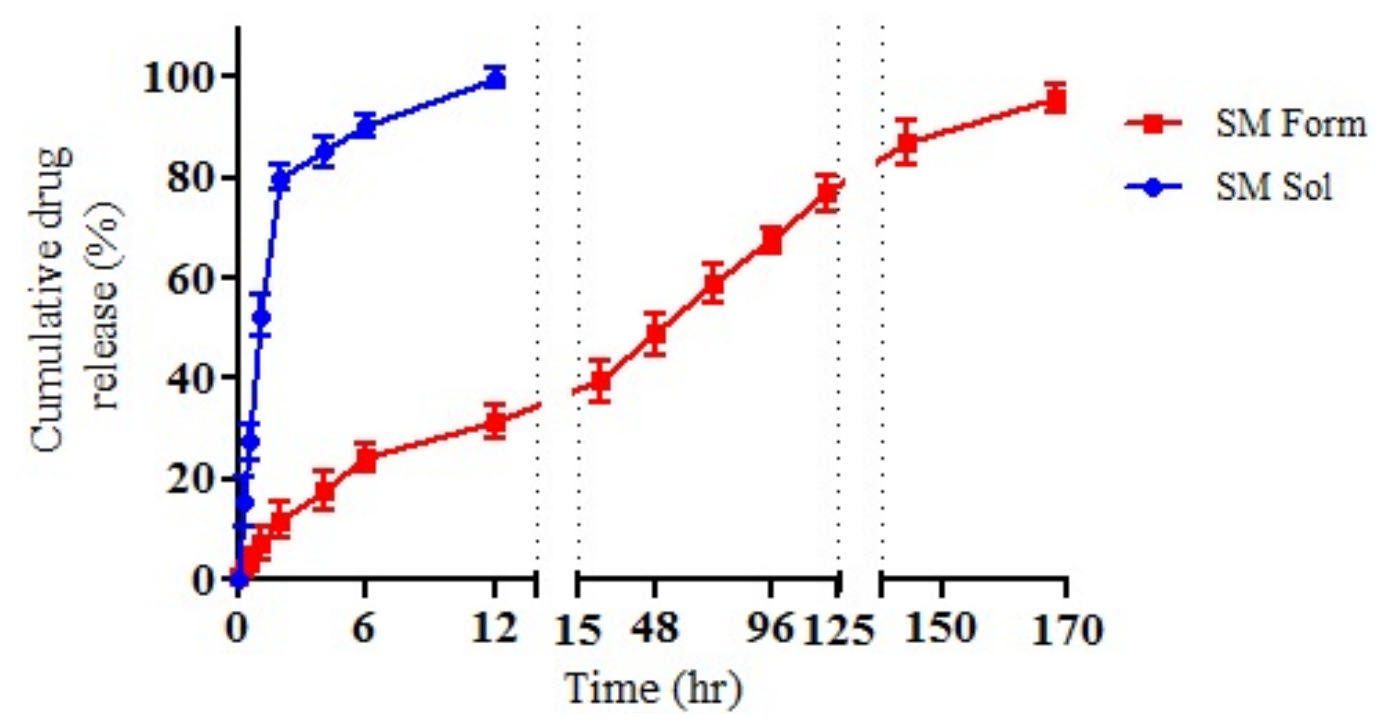

Figure 4. Drug release profile of sunitinib from the sunitinib solution and the SM-MPEG-PCL micelles formulation up to seven days at $37^{\circ} \mathrm{C}$ in a phosphate buffer at $\mathrm{pH} 7.4$ (mean $\pm \mathrm{SD}, n=3$ ). Dotted line represents gap in the continuous axis.

\subsection{Cytotoxicity Study}

The SM-MPEG-PCL, blank MPEG-PCL, and SM drug solution were evaluated for cytotoxicity by the use of an MTT assay performed on ARPE-19, a human retinal epithelium cell line, as seen in Figure 5. Cells were first treated with an incomplete medium and distributed according to concentrations of 0.001 , $0.01,0.1,1,10$, and $20 \mu \mathrm{M}$ for each formulation in quadruplicate for 24 and $48 \mathrm{~h}$. The cell viabilities of the formulations were compared to the positive control—a complete medium that obtained a standard percentage of $100 \%$ viability. It was concluded that the cell viability of the SM-MPEG-PCL and blank MPEG-PCL micelles was greater than $88 \%$ for all respective concentrations. The optimal cell viability percentage in the SM-MPEG-PCL formulation at $24 \mathrm{~h}$ was observed at a both concentrations of 10 and $20 \mu \mathrm{M}$, whereas in the blank micelles, the highest viability was at the concentrations of 0.01 and $0.1 \mu \mathrm{L}$ at a viability percentage of $99.25 \%$ (Figure $5 \mathrm{~A}$ ). The viability of the drug solution was $68 \%$ for $24 \mathrm{~h}$ and $61 \%$ at $48 \mathrm{~h}$, suggesting that the solution was toxic to the cells in higher concentrations.

The results for $48 \mathrm{~h}$ also showed that the SM-MPEG-PCL formulation and the blank micelles were viable, with percentages above $95 \%$ for all six concentrations (Figure 5B). The MTT assay analysis reaffirmed the SM-MPEG-PCL and blank micellar formulations' ability to show no significant cytotoxicity within the ARPE-19 cell line, thus indicating that the formulation was suitable for further comprehensive studies. 


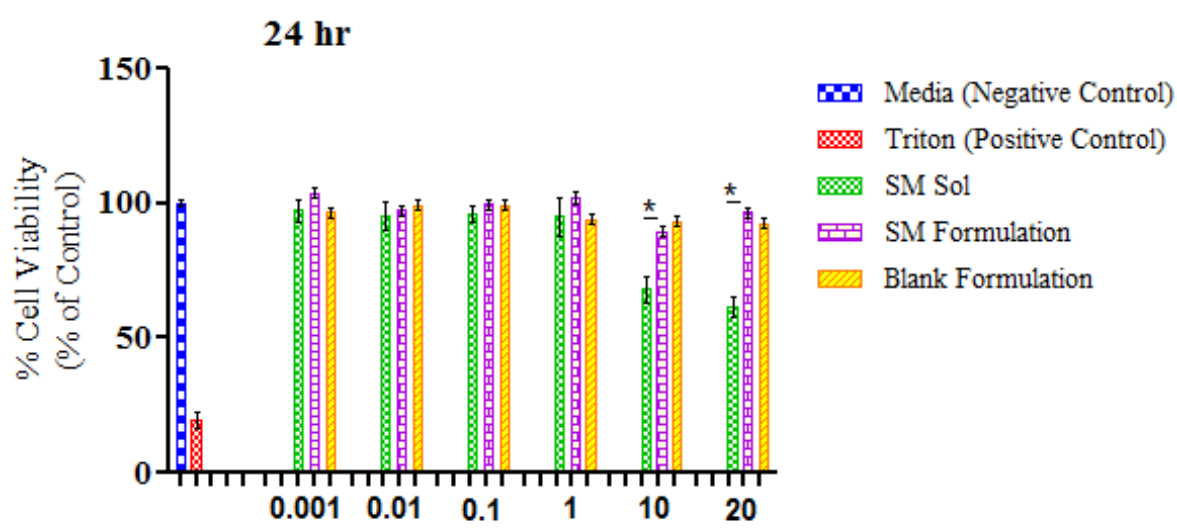

Concentration $(\mu \mathrm{M}) \quad * \mathrm{p}<0.01$

(A)

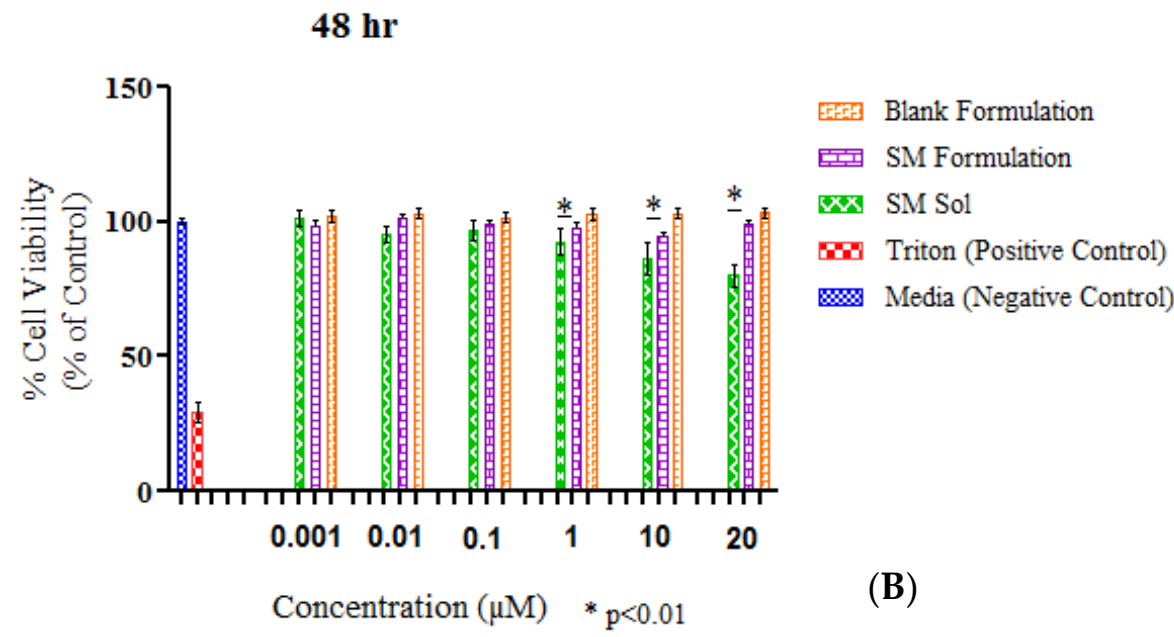

Figure 5. Percent cell viability at various concentrations of the sunitinib drug solution and the SMMPEG-PCL formulation at (A) 24 and (B) $48 \mathrm{~h}$ on the ARPE-19 human retinal cell line (mean \pm SD, $n=4)$.

\subsection{Anti-VEGF ELISA}

An anti-VEGF ELISA was used to determine the percentage of VEGF expression in vitro in cells after treatment with the SM drug solution and the SM-MPEG-PCL micelles. Following initial treatment and incubation, samples were obtained at time intervals of 24,48 , and $72 \mathrm{~h}$. These samples were then analyzed by the aid of a human VEGF-A ELISA kit. It was seen that VEGF expression decreased in the 24,48 , and $72 \mathrm{~h}$ periods for the free SM drug solution, whereas the SM-MPEG-PCL formulation also decreased the overall VEGF expression $(p<0.05)$ across 24,48 , and $72 \mathrm{~h}$ of exposure to formulations loaded with an equivalent $10 \mu \mathrm{M}$ drug concentration (Figure 6). In the anti-VEGF ELISA, the drug control was added to compare the inhibition effects of VEGF expression with pure drug and drug-loaded formulations. In both the cases, sunitinib malate was present, which is an anti-angiogenic drug that is responsible for reducing VEGF expression, in the cell wells. Meanwhile, the polymer was not found to exert an anti-angiogenic effect, and it was found to have no role in reducing VEGF expression. Thus, only the polymer control was not added to the anti-VEGF ELISA. However, a polymer control as a blank formulation was added to the MTT assay to evaluate the toxicity of the polymer itself. 


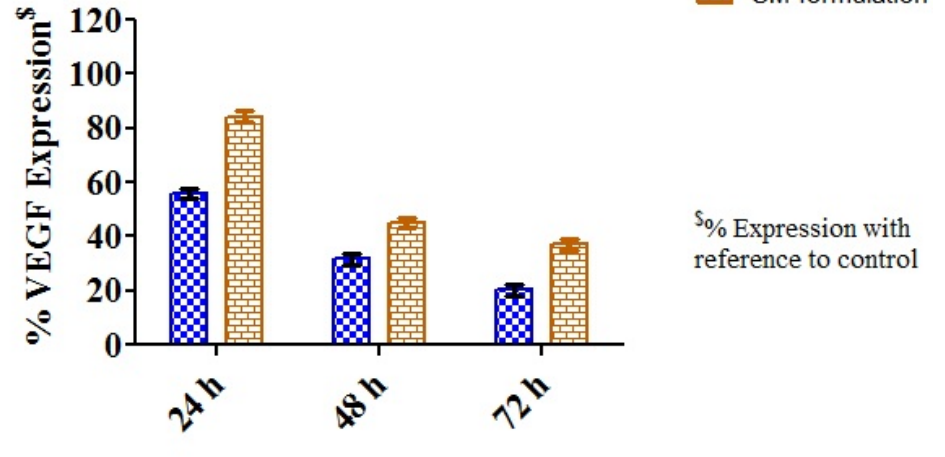

Concentration $(\mu \mathrm{M})$

Figure 6. Percent vascular endothelial growth factor (VEGF) expression in ARPE-19 cells treated with the sunitinib drug solution and the MPEG-PCL formulation at 24, 48, and $72 \mathrm{~h}$ using ELISA (mean \pm SD, $n=4)$.

Based on in vitro drug release studies, $100 \%$ of the drug was available for exerting effect in the group of the drug solution, where it was completely released within $24 \mathrm{~h}$. While in the formulation, only $40-50 \%$ of the drug was available for activity, as at $48 \mathrm{~h}$, the released drug from micelles was around $40-50 \%$. Thus, such results of the anti-VEGF ELISA study were because of the availability of the drug for action after 24 and $48 \mathrm{~h}$. Moreover, the elimination half-life of drug is around 40-60 h. Thus, at $72 \mathrm{~h}$, the same pattern of results was seen due to the effectiveness of the already-released drug. This can be attributed to the need for a longer period of observation, which was indicative of the repetition of the experiment for a longer period of time. An observation period of $144 \mathrm{~h}$ sufficed in showing an increase in the SM free drug solution and, subsequently, a steady VEGF percentage decrease in the formulation. In summation, both the free SM drug solution and the SM-MPEG-PCL formulation showed an overall decrease in VEGF expression across a $24-72 \mathrm{~h}$ period. This suggested that the SM drug and the formulation are both suitable in decreasing overall expression of VEGF expression within ARPE-19 cells.

\section{Conclusions}

Sunitinib-loaded 5-2k MPEG-PCL micelles exhibited an average size of $134.2 \pm 2.3 \mathrm{~nm}$, an average zeta potential of $-0.159 \pm 0.07 \mathrm{mV}$, and a PDI of $0.160 \pm 0.08$. With an entrapment efficiency of $64.3 \pm 2.6 \%$, the SM-MPEG-PCL formulation was studied for biocompatibility within ARPE-19, a human retinal pigment epithelial cell line, to demonstrate its biological interactions in vitro to treat symptoms caused by AMD. The SM-MPEG-PCL formulation exhibited a sustained release profile at up to seven days, with an overall release percentage of $95.56 \pm 2.7 \%$. The SM-MPEG-PCL micelles showed a percent viability of above $88 \%$ for all six concentrations for $24 \mathrm{~h}$ and above $95 \%$ for $48 \mathrm{~h}$, thus demonstrating that the SM-MPEG-PCL formulation and the blank MPEG-PCL micelles were not toxic to the cells. The anti-VEGF ELISA confirmed that the drug solution and the formulation were effective at reducing the overall expression of the VEGF protein in the ARPE-19 human retinal pigment epithelial cell line across a 24-72 $\mathrm{h}$ period. In summation, the SM-MPEG-PCL micelles demonstrated an exceptional preliminary performance that is to be further studied in vivo to see if they can provide a more effective treatment for AMD.

Author Contributions: V.S. and P.B. conceived and designed the experiments; J.S. performed the experiments; P.B. and D.B. analyzed the data; V.S. and D.B. contributed reagents/materials/analysis tools; J.S. and P.B. wrote the paper. All authors have read and agreed to the published version of the manuscript.

Funding: This research received no external funding. 
Acknowledgments: The authors would also like to acknowledge the Lisa Muma Weitz Laboratory for Advanced Microscopy \& Cell Imaging, USF Health, University of South Florida, Tampa, FL, USA for providing facility for microscopy and Department of Chemistry, College of Arts and Science, University of South Florida, Tampa, FL, USA for providing facility for DSC study.

Conflicts of Interest: There is no conflict of interest.

\section{References}

1. Age-Related Macular Degeneration (AMD) Data and Statistics. Available online: https: //www.nei.nih.gov/learn-about-eye-health/resources-for-health-educators/eye-health-data-and-stat istics/age-related-macular-degeneration-amd-data-and-statistics (accessed on 10 September 2019).

2. Pennington, K.L.; DeAngelis, M.M. Epidemiology of age-related macular degeneration (AMD): Associations with cardiovascular disease phenotypes and lipid factors. Eye Vis. 2016, 3, 34. [CrossRef] [PubMed]

3. Lim, L.S.; Mitchell, P.; Seddon, J.M.; Holz, F.G.; Wong, T.Y. Age-related macular degeneration. Lancet 2012, 379, 1728-1738. [CrossRef]

4. Bhatt, P.; Kelly, S.; Sutariya, V. Nanoscale delivery systems in treatment of posterior ocular neovascularization: Strategies and potential applications. Ther. Deliv. 2019, 10, 737-747. [CrossRef] [PubMed]

5. Budzinskaya, M.V.; Plyukhova, A.A.; Sorokin, P.A. Anti-VEGF therapy resistance in neovascular age-related macular degeneration. Vestn. Oftalmol. 2017, 133, 103-108. [CrossRef]

6. van der Giet, M.; Henkel, C.; Schuchardt, M.; Tolle, M. Anti-VEGF Drugs in Eye Diseases: Local Therapy with Potential Systemic Effects. Curr. Pharm. Des. 2015, 21, 3548-3556. [CrossRef]

7. Le Tourneau, C.; Raymond, E.; Faivre, S. Sunitinib: A novel tyrosine kinase inhibitor. A brief review of its therapeutic potential in the treatment of renal carcinoma and gastrointestinal stromal tumors (GIST). Ther. Clin. Risk Manag. 2007, 3, 341-348. [CrossRef]

8. Mena, A.C.; Pulido, E.G.; Guillén-Ponce, C. Understanding the molecular-based mechanism of action of the tyrosine kinase inhibitor: Sunitinib. Anti-Cancer Drugs 2010, 21, S3-S11. [CrossRef]

9. Brossa, A.; Grange, C.; Mancuso, L.; Annaratone, L.; Satolli, M.A.; Mazzone, M.; Camussi, G.; Bussolati, B. Sunitinib but not VEGF blockade inhibits cancer stem cell endothelial differentiation. Oncotarget 2015, 6, 11295-11309. [CrossRef]

10. Roskoski, R., Jr. Sunitinib: A VEGF and PDGF receptor protein kinase and angiogenesis inhibitor. Biochem. Biophys. Res. Commun. 2007, 356, 323-328. [CrossRef]

11. Papaetis, G.S.; Syrigos, K.N. Sunitinib: A multitargeted receptor tyrosine kinase inhibitor in the era of molecular cancer therapies. BioDrugs Clin. Immunother. Biopharm. Gene Ther. 2009, 23, 377-389. [CrossRef]

12. Hao, Z.; Sadek, I. Sunitinib: The antiangiogenic effects and beyond. OncoTargets Ther. 2016, 9, 5495-5505. [CrossRef] [PubMed]

13. Varano, M.; Eter, N.; Winyard, S.; Wittrup-Jensen, K.U.; Navarro, R.; Heraghty, J. Current barriers to treatment for wet age-related macular degeneration (wAMD): Findings from the wAMD patient and caregiver survey. Clin. Ophthalmol. 2015, 9, 2243-2250. [CrossRef] [PubMed]

14. Vadlapudi, A.D.; Mitra, A.K. Nanomicelles: An emerging platform for drug delivery to the eye. Ther. Deliv. 2013, 4, 1-3. [CrossRef] [PubMed]

15. Cholkar, K.; Patel, A.; Vadlapudi, A.D.; Mitra, A.K. Novel Nanomicellar Formulation Approaches for Anterior and Posterior Segment Ocular Drug Delivery. Recent Pat. Nanomed. 2012, 2, 82-95. [CrossRef]

16. Grossen, P.; Witzigmann, D.; Sieber, S.; Huwyler, J. PEG-PCL-based nanomedicines: A biodegradable drug delivery system and its application. J. Control. Release Off. J. Control. Release Soc. 2017, 260, 46-60. [CrossRef]

17. Mandal, A.; Bisht, R.; Rupenthal, I.D.; Mitra, A.K. Polymeric micelles for ocular drug delivery: From structural frameworks to recent preclinical studies. J. Control. Release Off. J. Control. Release Soc. 2017, 248, 96-116. [CrossRef]

18. Danafar, H. MPEG-PCL copolymeric nanoparticles in drug delivery systems. Cogent Med. 2016. [CrossRef]

19. Cuong, N.-V.; Jiang, J.-L.; Li, Y.-L.; Chen, J.-R.; Jwo, S.-C.; Hsieh, M.-F. Doxorubicin-Loaded PEG-PCL-PEG Micelle Using Xenograft Model of Nude Mice: Effect of Multiple Administration of Micelle on the Suppression of Human Breast Cancer. Cancers 2010, 3, 61-78. [CrossRef]

20. Shi, S.; Peng, F.; Zheng, Q.; Zeng, L.; Chen, H.; Li, X.; Huang, J. Micelle-solubilized axitinib for ocular administration in anti-neovascularization. Int. J. Pharm. 2019, 560, 19-26. [CrossRef] 
21. Torchilin, V.P. Micellar nanocarriers: Pharmaceutical perspectives. Pharm. Res. 2007, 24, 1-16. [CrossRef]

22. Al-Zamil, W.M.; Yassin, S.A. Recent developments in age-related macular degeneration: A review. Clin. Interv. Aging 2017, 12, 1313-1330. [CrossRef] [PubMed]

23. Del Amo, E.M.; Urtti, A. Current and future ophthalmic drug delivery systems. A shift to the posterior segment. Drug Discov. Today 2008, 13, 135-143. [CrossRef] [PubMed]

24. Booth, B.A.; Vidal Denham, L.; Bouhanik, S.; Jacob, J.T.; Hill, J.M. Sustained-release ophthalmic drug delivery systems for treatment of macular disorders: Present and future applications. Drugs Aging 2007, 24, 581-602. [CrossRef] [PubMed]

25. Rizzo, M.; Porta, C. Sunitinib in the treatment of renal cell carcinoma: An update on recent evidence. Ther. Adv. Urol. 2017, 9, 195-207. [CrossRef] [PubMed]

26. Patil, S.; Bhatt, P.; Lalani, R.; Amrutiya, J.; Vhora, I.; Kolte, A.; Misra, A. Low molecular weight chitosan-protamine conjugate for siRNA delivery with enhanced stability and transfection efficiency. RSC Adv. 2016, 6, 110951-110963. [CrossRef]

27. Bhatt, P.; Lalani, R.; Vhora, I.; Patil, S.; Amrutiya, J.; Misra, A.; Mashru, R. Liposomes encapsulating native and cyclodextrin enclosed paclitaxel: Enhanced loading efficiency and its pharmacokinetic evaluation. Int. J. Pharm. 2018, 536, 95-107. [CrossRef]

28. Yewale, C.; Baradia, D.; Patil, S.; Bhatt, P.; Amrutiya, J.; Gandhi, R.; Kore, G.; Misra, A. Docetaxel loaded immunonanoparticles delivery in EGFR overexpressed breast carcinoma cells. J. Drug Deliv. Sci. Technol. 2018, 45, 334-345. [CrossRef]

29. Patil, S.; Lalani, R.; Bhatt, P.; Vhora, I.; Patel, V.; Patel, H.; Misra, A. Hydroxyethyl substituted linear polyethylenimine for safe and efficient delivery of siRNA therapeutics. RSC Adv. 2018, 8, 35461-35473. [CrossRef]

30. Tandel, H.; Bhatt, P.; Jain, K.; Shahiwala, A.; Misra, A. In-vitro and in-vivo tools in emerging drug delivery scenario: Challenges and updates. In In-Vitro and In-Vivo Tools in Drug Delivery Research for Optimum Clinical Outcomes; Ambikanandan, M., Aliasgar, S., Eds.; CRC Press: Boca Raton, FL, USA, 2018.

31. Sharif Makhmal Zadeh, B.; Niro, H.; Rahim, F.; Esfahani, G. Ocular Delivery System for Propranolol Hydrochloride Based on Nanostructured Lipid Carrier. Sci. Pharm. 2018, 86, 16. [CrossRef]

32. Rini, B.I.; Melichar, B.; Fishman, M.N.; Oya, M.; Pithavala, Y.K.; Chen, Y.; Bair, A.H.; Grünwald, V. Axitinib dose titration: Analyses of exposure, blood pressure and clinical response from a randomized phase II study in metastatic renal cell carcinoma. Ann. Oncol. Off. J. Eur. Soc. Med Oncol. 2015, 26, 1372-1377. [CrossRef]

33. Cáceres, C.; Canfarotta, F.; Chianella, I.; Pereira, E.; Moczko, E.; Esen, C.; Guerreiro, A.; Piletska, E.; Whitcombe, M.J.; Piletsky, S.A. Does size matter? Study of performance of pseudo-ELISAs based on molecularly imprinted polymer nanoparticles prepared for analytes of different sizes. Analyst 2016, 141, 1405-1412. [CrossRef] [PubMed]

34. Bhatt, P.; Narvekar, P.; Lalani, R.; Chougule, M.B.; Pathak, Y.; Sutariya, V. An in vitro Assessment of Thermo-Reversible Gel Formulation Containing Sunitinib Nanoparticles for Neovascular Age-Related Macular Degeneration. AAPS PharmSciTech 2019, 20, 281. [CrossRef] [PubMed]

35. Clogston, J.D.; Patri, A.K. Zeta potential measurement. Methods Mol. Biol. 2011, 697, 63-70. [CrossRef] [PubMed]

36. Rogošić, M.; Mencer, H.J.; Gomzi, Z. Polydispersity index and molecular weight distributions of polymers. Eur. Polym. J. 1996, 32, 1337-1344. [CrossRef]

37. Qiu, J.F.; Gao, X.; Wang, B.L.; Wei, X.W.; Gou, M.L.; Men, K.; Liu, X.Y.; Guo, G.; Qian, Z.Y.; Huang, M.J. Preparation and characterization of monomethoxy poly(ethylene glycol)-poly( $\varepsilon$-caprolactone) micelles for the solubilization and in vivo delivery of luteolin. Int. J. Nanomed. 2013, 8, 3061-3069. [CrossRef]

38. Narvekar, P.; Bhatt, P.; Fnu, G.; Sutariya, V. Axitinib-Loaded Poly(Lactic-Co-Glycolic Acid) Nanoparticles for Age-Related Macular Degeneration: Formulation Development and In Vitro Characterization. ASSAY Drug Dev. Technol. 2019, 17, 167-177. [CrossRef]

(C) 2020 by the authors. Licensee MDPI, Basel, Switzerland. This article is an open access article distributed under the terms and conditions of the Creative Commons Attribution (CC BY) license (http://creativecommons.org/licenses/by/4.0/). 